\title{
Multiple deep levels in metalorganic vapor phase epitaxy GaAs grown by controlled oxygen incorporation
}

\author{
J. W. Huang and T. F. Kuech \\ Department of Chemical Engineering, University of Wisconsin, Madison, Wisconsin 53706
}

(Received 21 March 1994; accepted for publication 17 May 1994)

\begin{abstract}
Oxygen intentionally introduced into GaAs, grown through the metalorganic vapor phase epitaxy process, is shown to introduce a controlled series of deep levels. A novel, commercially available, oxygen precursor, $\left(\mathrm{C}_{2} \mathrm{H}_{5}\right)_{2} \mathrm{AlOC}_{2} \mathrm{H}_{5}$, was used as the oxygen source. Capacitance measurements have revealed compensation of both donors and acceptors in these materials when using this source. The determination of the deep level structure of these films has been carried out through the use of deep level transient spectroscopy (DLTS). The DLTS investigation, carried out on GaAs $p^{+}-n$ homojunctions, indicates that unlike the case of bulk oxygen-doped GaAs (GaAs:O), several deep levels are introduced and directly associated with the intentional oxygen introduction. Two principal traps are found to be located at 0.95 and $0.75 \mathrm{eV}$ below the conduction-band edge, with several other minor traps being observed. These intentionally introduced deep levels have been used to form highly resistive GaAs, providing an analog to the currently employed low-temperature semi-insulating nonstoichiometric GaAs grown by molecular beam epitaxy.
\end{abstract}

Semi-insulating (SI) substrates are generally used in the formation of GaAs-based metal-semiconductor field-effect transistor (MESFET) devices in order to reduce parasitic capacitance and eliminate device cross-talk. A number of significant technological problems are encountered in MESFET applications, both to digital and analog circuits, which are related to the SI substrates. These problems include sidegating and backgating effects, light sensitivity, low output resistance, and low source-drain breakdown voltage. ${ }^{1}$ An epilaxial buffer layer, providing higher resistivity and optical insensitivity, can be inserted between the active layer and the substrate to alleviate or even eliminate these problems. ${ }^{1,2}$ The controlled introduction of deep levels, in general, has long been recognized as a principal means of producing SI compound semiconductors. The choice of suitable chemical species for the fabrication of SI layers is, however, complicated by the constraints on the diffusion coefficient and solubility of the impurity, as well as the depth, within the band gap, of the level produced. Transition elements used in this application, ${ }^{3,4}$ for example, exhibit a high thermal diffusion coefficient and are not optimal for the formation of ionimplanted device structures.

Oxygen doping of GaAs has been recently investigated as a new alternative for the formation of metalorganic vapor phase epitaxially (MOVPE) based SI GaAs. ${ }^{5}$ Oxygen can be intentionally incorporated into GaAs through the independent introduction of aluminum-oxygen bonding based precursors; dimethyl aluminum methoxide [DMALO, $\left.\left(\mathrm{CH}_{3}\right)_{2} \mathrm{AlOCH}_{3}\right]^{5}$ or diethyl aluminum ethoxide [DEALO, $\left.\left(\mathrm{C}_{2} \mathrm{H}_{5}\right)_{2} \mathrm{AlOC}_{2} \mathrm{H}_{5}\right]^{6}{ }^{6}$ Oxygen-doped epitaxial layers have a specular surface morphology and high crystal quality. Oxygen concentrations ranging from $10^{15}$ to $10^{20} \mathrm{~cm}^{-3}$ can be controllably achieved by varying the growth conditions. Oxygen-related deep levels are generated, compensating shallow donors. The use of oxygen-doped GaAs for semiinsulating applications in GaAs will depend on the details of the deep level structure as well as the issues of uniformity, thermal stability, and reproducibility of oxygen incorporation. The thorough characterization needed to fully exploit the potential of this new material, however, has not been completed.

This letter addresses the nature of the deep level structure formed in GaAs using DEALO. The co-introduction of $\mathrm{Al}$ and $\mathrm{O}$ into the epitaxial GaAs leads to the formation of a multiple deep level structure which is significantly different from the previously studied case of bulk oxygen-doped GaAs (GaAs:O) ${ }^{7-9}$ The presence of two major deep level traps is rationalized in terms of the possible variations in the local bonding around the GaAs:O defect associated with the co-doping of $\mathrm{Al}$.

The primary oxygen source used here, in the MOVPE formation of GaAs:O, is DEALO, which offers advantages over DMALO. ${ }^{6}$ DEALO is a commercially available liquid at room temperature (melting point $\sim 2.5^{\circ} \mathrm{C}$ ) with suitable vapor pressure for use as a dopant, leading to a more reproducible oxygen incorporation. The DEALO was employed as received without additional purification. All samples were grown in a conventional horizontal low pressure (78 Torr) reactor, ${ }^{10}$ using trimethyl gallium (TMGa) and arsine $\left(\mathrm{AsH}_{3}\right)$. Disilane $\left(\mathrm{Si}_{2} \mathrm{H}_{6}\right)$ and carbon tetrachloride $\left(\mathrm{CCl}_{4}\right)$ were employed for $n$ - and $p$-type doping, respectively. The growth temperature was $600^{\circ} \mathrm{C}$ and the V/III ratio $\left(\mathrm{AsH}_{3} / \mathrm{TMGa}\right)$ was 40 . The growth rate was held constant at $0.05 \mu \mathrm{m} / \mathrm{min}$, corresponding to a TMGa mole fraction of $\sim 1.8 \times 10^{-4}$ in the reactor. The deep level transient spectroscopy (DLTS) measurements were carricd out on $p^{+}-n$ homojunctions. ${ }^{11}$ The homojunctions consisted of a $1-\mu \mathrm{m}$-thick $n$-type region capped by a $0.5-\mu \mathrm{m} p^{+}$layer doped with $4 \times 10^{18} \mathrm{~cm}^{-3}$ carbon, grown on Si-doped $n^{+}\langle 100\rangle$ substrates. These $p^{+}-n$ junctions were fabricated by a standard lithography and liftoff process. $\mathrm{Ge} / \mathrm{Ni} / \mathrm{Al}$ dots, $500 \mu \mathrm{m}$ in diameter, were deposited and alloyed for ohmic contact on $p^{+}$capping layer followed by mesa etching. In/Sn ohmic contact was then alloyed to the $n^{+}$substrate for back side ohmic contact. The $n$-type region was co-doped with DEALO and $\mathrm{Si}_{2} \mathrm{H}_{6}$ to facilitate the investigation of oxygen-induced defects by DLTS. Conventional DLTS measurements, using double boxcar correlators, were performed over the temperature 
TABLE I. DLTS samples were grown with disilane mole fraction $5 \times 10^{-9}$. DEALO mole fractions for samples 1 and 2 are $7.5 \times 10^{-7}$ and $1.5 \times 10^{-6}$, respectively. [Si], [Al], and [O] are estimated from SIMS results (Ref. 6). $N_{d}-N_{a}$ are determined by $E C-V$ profiling. All concentrations are in the unit of $1 \times 10^{17} \mathrm{~cm}^{-3}$.

\begin{tabular}{ccccccc}
\hline & & & \multicolumn{1}{c}{$N_{d}-N_{a}$} \\
Sample & {$[\mathrm{Si}]$} & {$[\mathrm{Al}]$} & {$[\mathrm{O}]$} & with DEALO & $\Delta\left(N_{d} N_{a}\right)$ & Compensation \\
\hline 1 & 1.06 & 3.2 & 0.01 & 0.944 & 0.116 & $10.94 \%$ \\
2 & 1.06 & 6.5 & 0.15 & 0.544 & 0.516 & $48.68 \%$ \\
\hline \hline
\end{tabular}

range of 77-425 K. Typical biasing and pulsing conditions are as follows: reverse bias $-1 \mathrm{~V}$, pulse height $1 \mathrm{~V}$, and pulse time width $1 \mathrm{~ms}$. Due to the high deep level concentration, constant-capacitance DLTS (CC-DLTS) measurements were also performed. The use of conventional DLTS measurements in highly defected materials can lead to nonexponential transients which complicate the data analysis. In CCDLTS, voltage transients are acquired, instead of the conventional capacitance transients, in order to eliminate ambiguity in the analysis attributable to the changing sampling volume. ${ }^{12}$ Our conventional DLTS measurements were therefore complemented by CC-DLTS to ensure the validity of our interpretation of the data.

While many samples were grown and characterized in this study, we will present results for two specific oxygen concentrations by using two different DEALO mole fraction conditions during the growth, while keeping $\mathrm{Si}_{2} \mathrm{H}_{6}$ mole fraction constant. The results described below were quite reproducible in terms of the doping levels and electrical behavior. Concentrations of $\mathrm{Si}, \mathrm{Al}$, and $\mathrm{O}$, as estimated from the results of secondary ion mass spectroscopy (SIMS) measurements, ${ }^{6}$ as well as electrochemical capacitancevoltage $(E C-V)$ profiling results are summarized in Table I. Oxygen concentrations were obtained by extrapolation since the SIMS detection limit for $O$ is $\sim 10^{17} \mathrm{~cm}^{-3}$. A low concentration of aluminum is also incorporated into the film along with a comparable or lower amount of oxygen. The EC-V profiling measurements indicate that the oxygen doping can introduce a controlled and reproducible level of compensation, defined here to be

$$
\frac{n_{\text {no doping }}-n_{\text {oxygen doping }}}{n_{\text {no doping }}} \times 100 \% .
$$

Typical DLTS spectra, obtained under conventional DLTS conditions, are shown in Fig. 1 for both the high and low oxygen concentration samples. CC-DLTS was also carried out on sample 2. The CC-DLTS measurements have produced identical results to the conventional DLTS spectra. Multiple deep levels were observed in both samples as compared to the reported single level in bulk GaAs:O, ${ }^{7}$ underlining the complexity of possible defect configurations due to the doping of DEALO and disilane. A comparison of the spectra in Fig. 1 indicates that the principal spectral features, peaks $\mathrm{D}$ and $\mathrm{E}$, are resolved in both samples. The Si concentration remained constant and the shift in the relative peak heights between these two samples should be related to the increased $\mathrm{Al}$ and $\mathrm{O}$ concentrations. The possible existence of peaks $A, B$, and $C$ in sample 1 cannot be confirmed since

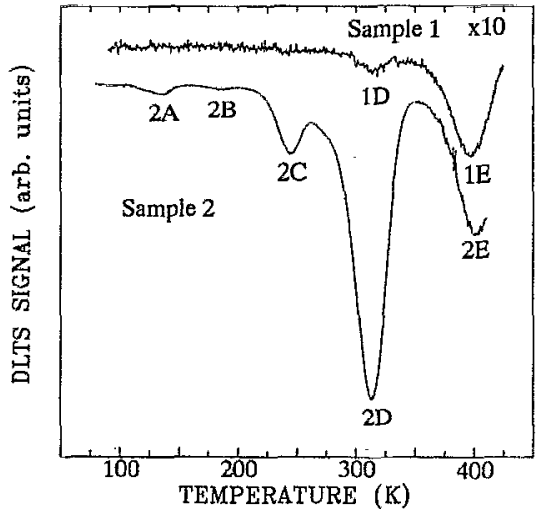

FIG. 1. DLTS spectra of DEALO and disilane co-doped GaAs. Multiple deep levels, with principal peaks at 0.75 and $0.95 \mathrm{eV}$ below $E_{c}$, are noted. Measurement conditions are as follows: reverse bias $-1 \mathrm{~V}$, pulse height $1 \mathrm{~V}$, pulse width $1 \mathrm{~ms}$, pulse period $500 \mathrm{~ms}$, and rate window $4.65 \mathrm{~s}^{-1}$.

they are beyond the detection limits. The Arrhenius plots of these emission peaks are shown in Fig. 2, and the DLTS measurement results from these two samples are summarized in Table II. We have noticed a change in $N_{d}-N_{a}$ between $E C-V$ results and $C-V$ measurements on $p^{+}-n$ junctions (Tables I and II). The origin of this observed difference is still unknown.

The quiescent reverse bias voltage was kept at $-1 \mathrm{~V}$ during the DLTS measurement. The amplitude of the filling pulse was limited to $+1 \mathrm{~V}$, ensuring that only majority carrier (electron) traps were detected in our measurement. This pulsing scheme was utilized since we have observed the controlled compensation of $p$-type carbon-doped $\mathrm{GaAs}$ by using DEALO. ${ }^{13}$ Pulsing into the forward bias region will lead to the injection of holes into $n$ region, where they could be trapped and re-emitted, complicating the data analysis. An additional quiescent reverse bias voltage value of $-2 \mathrm{~V}$ was used while the amplitude of the filling pulse remained the same. The relative peak heights and peak positions remained unchanged, indicating the presence of an uniform distribution of oxygen-related deep traps, agreeing with the $E C-V$

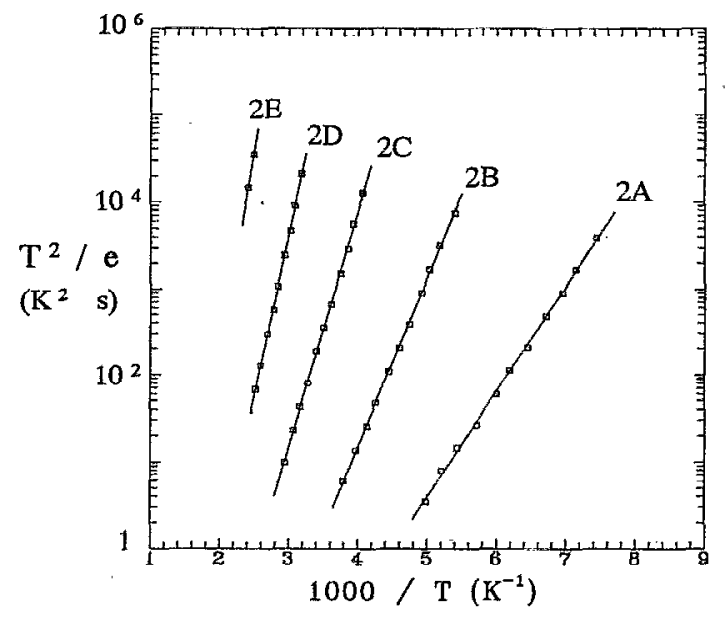

FIG. 2. The Arrhenius plots of sample 2 in Fig. 1 showing the signatures of electron traps. 
TABLE II. Deep level characteristics of samples 1 and 2 corresponding to the spectra in Fig. $1 . N_{d}-N_{a}$ are determined by $C-V$ measurement on $p^{+}-n$ junctions. Trap cross section areas $\sigma$ are obtained from intercepts of Arrhenius plots in Fig. 2.

\begin{tabular}{ccccccc}
\hline \hline Sample & $\begin{array}{c}N_{d}-N_{a} \\
\left(\times 10^{16} \mathrm{~cm}^{-3}\right)\end{array}$ & Trap & $\begin{array}{c}\Delta E_{t} \\
(\mathrm{eV})\end{array}$ & $\begin{array}{c}\sigma \\
\left(\times 10^{-14} \mathrm{~cm}^{2}\right)\end{array}$ & $\begin{array}{c}N_{t} \\
\left(\times 10^{14} \mathrm{~cm}^{-3}\right)\end{array}$ & $\begin{array}{c}\text { Total } N_{t} \\
\left(\times 10^{14} \mathrm{~cm}^{-3}\right)\end{array}$ \\
\hline 1 & 7.8 & 1D & 0.72 & 7.88 & 0.583 & 2.78 \\
& \multirow{2}{*}{1.7} & 1E & 0.94 & 12.7 & 2.2 & 56.54 \\
& 2A & 0.24 & 0.154 & 0.816 & \\
& 2B & 0.39 & 2.22 & 0.517 & \\
& 2C & 0.54 & 5.19 & 4.81 & \\
& 2D & 0.75 & 20.2 & 31.9 & \\
& 2E & 0.95 & 10.7 & 18.5 & \\
\hline \hline
\end{tabular}

profiles. ${ }^{6}$ Trap filling pulse time width during DLTS scan was set to be $1 \mathrm{~ms}$. This pulse duration was verified to be sufficient to fill greater than $95 \%$ of the available traps. Total trap concentrations, however, cannot account for all of the observed compensation from $E C-V$ results (Table I). Origins of this discrepancy are still unknown. Additional levels with very large emission time constants or neutral dopant-oxygen complexes could account for part of this discrepancy.

The presence of multiple deep level peaks in Fig. 1 can be rationalized by the analogy to the case of DX center in low $\mathrm{Al}$ content $\mathrm{Al}_{x} \mathrm{Ga}_{1-x}$ As. In the case of the Si-based DX center, multiple emission peaks were observed in samples containing a low amount of $\mathrm{Al}^{14}$ These multiple emission energies are related to the shift in the local environment of the DX center. At low Al concentrations, there is a variation in the local environment on the second nearest-neighbor shell (Si resides on a cation site) due to the random distribution of $\mathrm{Al}$ atoms on the cation sublattice. We have reported a linear and fourth-order dependence of the $\mathrm{Al}$ and $\mathrm{O}$ concentrations, respectively, on DEALO mole fraction in the reactor. $^{6}$ This growth observation indicates that multiple $\mathrm{Al}-\mathrm{O}$ based species are responsible for the physical incorporation of oxygen. The presence of multiple $\mathrm{Al}$ atoms locally associated with the oxygen atom in the lattice is therefore expected. The variations in local environment about the oxygen atom could lead to multiple DLTS emission peaks, each associated with a separate Al-O configuration. It should be mentioned that an alternative, and perhaps less likely explanation, for the multiple emission peaks would be the formation of a Si-O related defect. ${ }^{6}$ The growth of the low emission energy peaks, $A$ through $D$, with respect to peak $E$ in Fig. 1, is seen as the DEALO mole fraction is increased while that of $\mathrm{Si}_{2} \mathrm{H}_{6}$ kept constant. Peak E could possibly be assigned to a Si-O related defect. A strong Si-O bond, comparable to the Al-O bond would favor this defect formation. The very low concentration of $\mathrm{Si}$ in the crystal and in the gas phase, as well as the absence of any intentionally introduced $\mathrm{Si}-\mathrm{O}$ based precursor, makes this reaction improbable. The nonlinear incorporation of oxygen, with the change in the distribution of Al-O local configurations, independent of the Si concentration would seem at this point more likely. Changes in the DLTS spectra, with oxygen concentration, reflect the shift in distribution of these local configurations. Additional work is required to clarify and substantiate this model of the microscopic origin of the deep level energy structure.
In summary, a novel oxygen precursor, DEALO, was used as the oxygen source. Both the commercial availability and easy handling of DEALO have provided a convenient means for the controlled introduction of deep levels into GaAs. The observed strong compensation of shallow impurities by DEALO doping originates from these oxygen-based deep levels. The major deep electron traps from DLTS spectra, with ionization energy at 0.75 and $0.95 \mathrm{eV}$, are near the middle of the band gap, suggesting that this material is a promising candidate for the formation of semi-insulating GaAs. We have previously reported that resistivities of more than $2 \times 10^{9} \Omega \mathrm{cm}$ at $294 \mathrm{~K}$ can be achieved in DEALOdoped GaAs. ${ }^{15}$ An activation energy of $0.81 \mathrm{eV}$ was also deduced from temperature-dependent resistivity measurement. This material thus provides an analogy to the currently employed low-temperature semi-insulating nonstoichiometric GaAs grown by molecular beam epitaxy for electronic and, possibly, photonic applications.

The authors would like to acknowledge the technical discussion with K. L. Bray, and the support of the Army Research Office, the Naval Research Laboratory, and the National Science Foundation under DMR-9201558.

${ }^{1}$ F. W. Smith, A. R. Calawa, C. L. Chen, M. J. Manfra, and L. J. Mahoney, IEEE Electron Devices Lett. 9, 77 (1988).

${ }^{2}$ G. Strauch, D. Schmitz, M. Heyen, and H. Juergensen, in Proceedings of the 6th Conference on Semi-Insulating III-V Materials (Institute of Physics, Bristol, 1991), p. 89.

${ }^{3}$ S. J. Bass, J. Cryst. Growth 44, 29 (1978).

${ }^{4}$ J. A. Long, V. G. Riggs, and W. D. Johnston, Jr., J. Cryst. Growth 69, 10 (1984).

${ }^{5}$ M. S. Goorsky, T. F. Kuech, F. Cardone, P. M. Mooney, G. J. Scilla, and R. M. Potemski, Appl. Phys. Lett. 58, 1979 (1991).

${ }^{6} \mathrm{~J}$. W. Huang, D. F. Gaines, T. F. Kuech, R. M. Potemski, and F. Cardone, J. Electron. Mater. 23, 659 (1994).

${ }^{7}$ J. Lagowski, D. G. Lin, T. Aoyama, and H. C. Gatos, Appl. Phys. Lett. 44, 336 (1984).

${ }^{8}$ M. Skowronski, S. T. Neild, and R. E. Kremer, Appl. Phys. Lett. 57, 902 (1990).

${ }^{9}$ H. C. Alt, Phys. Rev. Lett. 65, 3421 (1990).

${ }^{10}$ T. F. Kuech, D. J. Wolford, E. Veuhotf, V. Deline, P. M. Mooney, R. Potemski, and J. Bradley, J. Appl. Phys. 62, 632 (1987).

${ }^{11}$ P. J. Wang, T. F. Kuech, M. A. Tischler, P. Mooney, G. Scilla, and F. Cardone, J. Appl. Phys. 64, 4975 (1988).

${ }^{12}$ R. Y. DeJule, M. A. Haase, D. S. Ruby, and G. E. Stillman, Solid-State Electron. 28, 639 (1985).

${ }^{13} \mathrm{~J}$. W. Huang and T. F. Kuech (unpublished).

${ }^{14}$ P. M. Mooney, J. Appl. Phys. 67, R1 (1990).

${ }^{15} \mathrm{~J}$. W. Huang and T. F. Kuech, Mater. Res. Soc. Symp. Proc. 325, 305 (1994). 